\title{
Analysis of Soil Water Retention Data Using Artificial Neural Networks
}

\author{
Sharad K. Jain ${ }^{1}$; Vijay P. Singh, F.ASCE²; and M. Th. van Genuchten ${ }^{3}$
}

\begin{abstract}
Many studies of water flow and solute transport in the vadose zone require estimates of the unsaturated soil hydraulic properties, including the soil water retention curve (WRC) describing the relationship between soil suction and water content. An artificial neural network (ANN) approach was developed to describe the WRC using observed data from several soils. The ANN approach was found to produce equally or more accurate descriptions of the retention data as compared to several analytical retention functions popularly used in the vadose zone hydrology literature. Given sufficient input data, the ANN approach was also found to closely describe the hysteretic behavior of a soil, including observed scanning wetting and drying curves.
\end{abstract}

DOI: 10.1061/(ASCE)1084-0699(2004)9:5(415)

CE Database subject headings: Neural networks; Soil water; Soil water storage; Hysteresis; Soil suction.

\section{Introduction}

The soil water retention curve (WRC) describes the ability of a soil to store water at different suctions. This curve, also known as the soil moisture characteristic, is one of the most basic water properties of a soil relating soil suction (or the matric or pressure head) $h$, with the volumetric content $\theta$. As the suction increases, progressively smaller pores lose their water and hence the water content decreases.

The WRC of a soil depends upon both soil texture and soil structure. The amount of water retained at relatively low suctions depends primarily upon capillary effects and the soil pore-size distribution, and hence is strongly affected by the soil structure. Water retention at higher suctions is increasingly due to adsorption, and hence is affected more by soil texture and the specific surface area of a soil, as opposed to soil structure. Coarsetextured soils generally release their water much quicker than fine-textured soils which often have a much broader pore-size distribution so that when suction is increased, the water content will decrease only gradually. Hence, a coarse-textured (sandy) soil is able to retain less water as compared to a fine-textured (clayey) soil.

One complication in the description of the soil hydraulic properties is the hysteretic nature of the water retention function, i.e., the curve follows different paths depending upon the drying and wetting history of the soil. During wetting when the suction is gradually lowered, the water content is generally higher than at

\footnotetext{
${ }^{1}$ Scientist 'F', National Institute of Hydrology, Roorkee 247667, India.

${ }^{2}$ A. K. Barton Professor, Dept. of Civil and Environmental Engineering, Louisiana State Univ., Baton Rouge, LA 70803-6405. E-mail: cesing@1su.edu

${ }^{3}$ Soil Physicist, USDA-ARS Salinity Laboratory, 450 W. Big Springs Rd., Riverside, CA 92507-4617.

Note. Discussion open until February 1, 2005. Separate discussions must be submitted for individual papers. To extend the closing date by one month, a written request must be filed with the ASCE Managing Editor. The manuscript for this paper was submitted for review and possible publication on March 25, 2003; approved on January 7, 2004. This paper is part of the Journal of Hydrologic Engineering, Vol. 9, No. 5, September, 2004. CASCE, ISSN 1084-0699/2004/5-415-420/\$18.00.
}

the same suction during drying. Fig. 1 shows the hysteretic nature of the WRC for Caribou silt loam using data determined by Topp (1971) as tabulated by Mualem (1974) in his unsaturated soil hydraulic property catalogue. Notice that in addition to the main hysteretic loop, one also obtains secondary scanning curves as reflected by the smaller loops in Fig. 1.

Several theories have been advanced to explain the hysteretic nature of soils, including the independent domain theory (Everett 1955) and various modifications thereof (e.g., Topp 1971). The main causes of hysteresis (Hillel 1971) are (1) the "inkbottle" effect due to geometric nonuniformity of individual pores; (2) the contact angle effect; (3) aggregate impacts of such phenomena as swelling, shrinking, or aging; and (4) the effect of entrapped air pockets in soils that connect different size pores during wetting. Water retention data are often conveniently described using analytical expressions. A large number of equations have been proposed for this purpose (e.g., van Genuchten and Nielsen 1985; Leij et al. 1997), the more popular ones being those by Brooks and Corey (1964) and van Genuchten (1980). The Brooks and Corey (1964) WRC model is given by

$$
\begin{aligned}
\theta & =\theta_{r}+\left(\theta_{s}-\theta_{r}\right)(\alpha h)^{-\lambda} \quad \alpha h \geqslant 1 \\
& =\theta_{s} \quad \alpha h<1
\end{aligned}
$$

where $h=$ pressure head; $\theta=$ volumetric water content; $\theta_{s}=$ saturated water content; $\theta_{r}=$ residual water content; $\alpha=$ parameter whose inverse $\left(h_{a}=\alpha^{-1}\right)$ is frequently referred to as the air entry value; and $\lambda$ is sometimes named the pore-size distribution index. van Genuchten (1980) proposed an alternative S-shaped model for the retention curve as follows:

$$
\theta=\theta_{r}+\left(\theta_{s}-\theta_{r}\right)\left[1+|\alpha h|^{n}\right]^{-m}
$$

where $\alpha, n$ and $m=$ empirical constants defining the shape of the curve.

Recently, a two-parameter equation for water retention curve was proposed by Assouline et al. (1998)

$$
\theta=\theta_{L}+\left(\theta_{s}-\theta_{L}\right)\left\{1-\exp \left[-\xi\left(h^{-1}-h_{L}^{-1}\right)^{\eta}\right]\right\} \quad 0 \leqslant h \leqslant h_{L}
$$

where $h_{L}=$ suction head that corresponds to a very low water content $\theta_{L}$, where the hydraulic conductivity becomes negligible; 


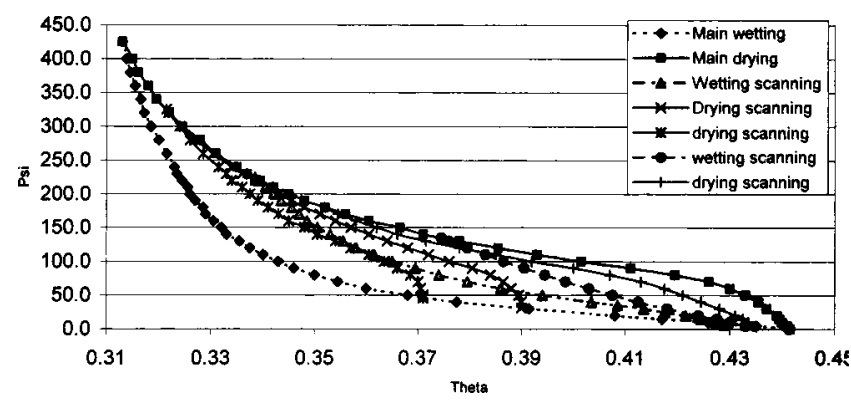

Fig. 1. Hysteretic water retention curves for Caribou silt loam (data from Topp 1971)

and $\xi$ and $\eta=$ empirical shape parameters. This model was tested on water retention data sets of 12 soils representing a range of soil textures from sand to clay. Agreement between the fitted curves and the measured data was reported to be the same or better than for other models.

One disadvantage of analytical expressions, such as those given by Eqs. (1)-(3), is that they are essentially empirical and incorporate certain assumptions about the shape of the WRC, including, for example the existence of a residual water contents in Eqs. (1) and (2). For this purpose several investigators have used alternative approaches to mathematically describe observed retention data, such as cubic splines (Kastanek and Nielsen 2001) or other more flexible functions (Prunty and Casey 2002). An emerging modeling technique that may be very well suited for this purpose is the artificial neural networks (ANNs). This empirical technique is now being applied successfully to a wide range of applications in hydrology. It is this technique that we will explore in this paper in an attempt to obtain improved descriptions of the water retention curve.

The main purpose of this paper hence is to use ANNs to describe the water retention curve of soils using measured soil water retention and suction data. Results will be compared with those using Eqs. (1), (2), and (3). We also apply the ANN model to a case with hysteresis involving drying and wetting curves and scanning curves. Below we first briefly review the use of ANNs in water resources.

\section{Artificial Neural Network Applications in Water Resources}

Artificial neural networks refer to computing systems whose central theme is borrowed from the analogy of biological neural networks. They represent highly simplified mathematical models of biological neural networks. They include the ability to learn and generalize from examples to produce meaningful solutions to problems even when input data contain errors or are incomplete, and to adapt solutions over time to compensate for changing circumstances and to process information rapidly.

The ANN approach is faster compared to its conventional counterparts, robust in noisy environments, and flexible in the range of problems it can solve. An ANN has the ability to learn from examples, to recognize a pattern in the data, to adapt solutions, and process information rapidly. Due to these advantages, ANNs have been used in numerous real world applications, such as image processing, speech processing, performing general mapping from input pattern to output pattern, and grouping similar patterns. Applications of ANNs to hydrology are rapidly gaining popularity due to their power and potential in mapping nonlinear system data.
A system may be nonlinear and multivariate, and the variables involved may have complex inter-relationships. Artificial neural networks are capable of adapting their complexity and their accuracy increases as more and more input data are made available to them. They are capable of extracting the relation between the input and output of a process without any knowledge of the underlying principles. Because of the generalizing capabilities of the activation function, one need not make any assumption about the relationship (linear or nonlinear) between input and output. All these properties make ANNs an attractive tool for water resources practitioners.

In the field of water resources, ANNs have been used for flow predictions, flow/pollution simulation, parameter identification, and to model complex nonlinear input-output time series. Hsu et al. (1995) have shown that the ANN approach provides a better representation of the rainfall-runoff relationship of a medium sized basin than does the ARMAX approach or the Sacramento soil moisture model. Raman and Sunilkumar (1995) investigated the use of ANNs for synthetic inflow generation and compared the model performance with that of a multivariate time series autoregressive moving average model. Minns and Hall (1996) applied an ANN to rainfall-runoff modeling. Dawson and Wilby (1998) used an ANN for river flow forecasting. Artificial neural networks were used for reservoir inflow prediction by Jain et al. (1999). Birikundavyi et al. (2002) found that an ANN can achieve accuracy superior to that of ARMAX and deterministic models for 7-day ahead forecasting. Kumar et al. (2002) concluded that the ANN can predict reference crop evapotranspiration for an area better than the Penman-Monteith method.

A set of two papers published by the ASCE task committee on application of ANNs in hydrology (ASCE 2000a, 2000b) contains a detailed review of the theory and applications of ANNs in water resources. Govindaraju and Rao (2000) have described many applications of ANNs to water resources.

Artificial neural networks have also recently found widespread application to predictions of the water retention curve from soil texture and related data using pedotransfer functions (PTFs) (e.g., Pachepsky et al. 1996; Schaap et al. 1998; Koekkoek and Booltink 1999; Minasny and McBratney 2002). The basic premise of these approaches is to assume the applicability of a certain retention model [e.g., Eqs. (1) or (2)], and then to use ANNs to correlate several or all of the unknown model parameters to more readily available or more easily measured data, such as soil texture, bulk density, organic matter content, and/or soil structure. As an example, Schaap et al. (1998) used ANNs to estimate the parameters in Eq. (2), as well as the saturated hydraulic conductivity. To facilitate the practical use of PTFs, they designed a hierarchical structure to allow input of both limited and more extended sets of predictors. They combined their ANNs with the bootstrap method (Efron and Tibshirani 1993) to additionally obtain an estimate of the uncertainty in the PTF predictions (Schaap et al. 1998). The PTFs were calibrated on some 2,100 soil hydraulic data sets, and further tested on more than 47,000 records from the Natural Resources Conservation Services soil characterization database. Schaap and Leij (2000) subsequently expanded the hierarchical approach to include unsaturated hydraulic conductivities. Their work resulted in a windows-based software package, Rosetta 〈http://www.ussl.ars.usda.gov/models/rosetta/ rosetta.htm $\rangle$ that incorporates the different PTFs.

An advantage of the PTF approach is that estimates of the complete retention function are obtained. Still, the predictions always remain somewhat approximate since they are based on generic, not site-specific data. If site-specific data are available, 


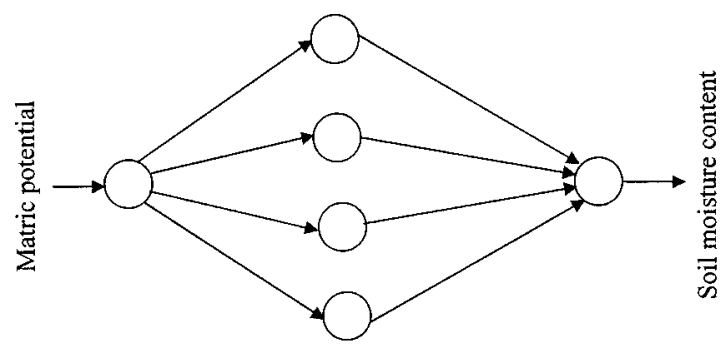

Input Layer

Hidden Layer

Output Layer

Fig. 2. Three-layer feed forward artificial neural network with one input node (for matric potential), four nodes in hidden layer, and one output node (representing soil moisture content)

several approaches may be used to obtain accurate descriptions of the retention data. This includes: (1) fitting the data with one of the empirical retention functions mentioned earlier; (2) using cubic splines (Kastanek and Nielsen 2001) or other methods for interpolation between measured retention data; and (3) using ANNs for the interpolation. It is the latter approach that is followed in this paper.

\section{Development of Artificial Neural Network Model and Analysis of Data}

A three-layer feed forward ANN was used in this study. According to Hsu et al. (1995), three-layer feed forward ANNs can be used to model real-world functional relationships that may be of unknown or poorly defined form and complexity. Such an ANN is shown in Fig. 2. The input to the network is received by the neurons in the input layer. The data passing through the connections from one neuron to another are manipulated by weights which control the strength of a passing signal. When these weights are modified, the data transferred through the network changes and the network output alters. The neurons in a layer share the same input and output connections, but do not interconnect among themselves. Each layer performs specific functions. All the nodes within a layer act synchronously, meaning at any point of time they will be at the same stage of processing. The level of activity generated at the output node(s) is the network's response to the inputs presented to it.

In this study, we applied the ANN results to the same data sets as used in the recent study by Assouline et al. (1998). The same data were also previously used by van Genuchten and Nielsen (1985) in their comparisons of the relative accuracy of the Brooks and Corey (1964) and van Genuchten (1980) retention models. All data sets were taken from the hydraulic property catalogue of Mualem (1974). Details of the data sets are given in Table 1. van Genuchten and Nielsen (1985) and Assouline et al. (1998) both fitted the WRC model parameters to the data using an iterative nonlinear regression procedure based on the MarquardtLevenburg algorithm (Marquardt 1963). Following Assouline et al. (1998), we compared the results of different approaches using the norm index (NI) defined as

$$
\mathrm{NI}=\sqrt{\sum_{i=1}^{n}\left(\theta_{o i}-\theta_{c i}\right)^{2}}
$$

Table 1. Soil Data Sets and Mualem (1974) Curve Types Used in This Study

\begin{tabular}{lcc}
\hline Soil type & Catalog number & Curve type \\
\hline Beit Netofa clay soil & 1006 & First drying \\
Rideau clay loam & 3101 & Main drying \\
Touchet silt loam & 3304 & First drying \\
Pachappa loam & 3403 & Main drying \\
Rubicon sandy loam & 3501 & Main drying \\
Pachappa fine sandy clay & 3503 & Main drying \\
Sable de riviere & 4118 & Main drying \\
\hline
\end{tabular}

where $\theta_{o i}$ and $\theta_{c i}=$ observed and computed values of the water content for the $i$ th observation, respectively.

A three-layer feed-forward ANN was trained for each data set. The input layer had one neuron that received matric potential values as input. The signal from the only neuron in the output layer represented the corresponding moisture content. The number of neurons in the hidden layer was determined by trial and error; five neurons gave the best results for the data used in this study. The sigmoid transfer function was used. This function is

$$
y_{j}=1 /\left[1+\exp \left(-z_{i}\right)\right]
$$

where $z_{i}=\Sigma w_{i j} x_{i} ; w_{i j}=$ weight of the connection from the $i$ th neuron in the previous layer to the current neuron; and $x_{i}=$ input to ith neuron in the previous layer. The sigmoid function is continuously differentiable. Its derivative is $y^{\prime}=y(1-y)$.

The weights of the ANN were estimated using an error backpropagation method. In this algorithm, a set of inputs and outputs was selected from the training set and the network calculated the output based on the inputs. The actual output was compared with the target output to find the output-layer errors. The weights of all neurons were adjusted based on the strength of the signal in the connection and the total measure of the error. The total error at the output layer was then reduced by redistributing this error backwards through the hidden layers until the input layer was reached. This process continued for a number of prescribed iterations or until a prescribed error tolerance was reached.

The mean square error (MSE) over the training samples is a typical objective function and can be expressed as

$$
E=\sum_{p=1}^{N} \sum_{n=1}^{m}\left(T_{p n}-O_{p n}\right)^{2}
$$

where $T_{p n}=$ target (or observed) value of the $n$th neuron for the $p$ th pattern; $O_{p n}=$ output value of the $n$th neuron for the $p$ th pattern; $N=$ total number of training patterns (sets of matric potential and soil moisture content values); and $m=$ total number of output neurons. About 10,000 iterations were performed to train the ANN for each data set.

The number of water retention $\theta-h$ data pairs for the main drying curves of the soil used in this study ranged from 14 to 34 . Before training, the $\theta$ values were normalized by using the following formula:

$$
\theta_{N}=\left(\theta-\theta_{\min }\right) / \Delta \theta
$$

where $\theta_{\min }=$ value slightly less than the minimum of the $\theta$ values; and $\Delta \theta=$ difference between the minimum and the maximum values. A different formula will be more suitable for a variable that varies within a certain range. Minns and Hall (1996) have rightly emphasized the importance of the correct standardization.

The suction values were normalized by dividing by the maximum $h$ value. This, however, caused a problem in the ANN train- 
Table 2. Comparison of Norm Index for Various Soils

\begin{tabular}{|c|c|c|c|c|c|c|}
\hline \multirow[b]{2}{*}{ Soil type } & \multicolumn{6}{|c|}{ Norm index obtained by } \\
\hline & $\begin{array}{l}\text { Catalog } \\
\text { number }\end{array}$ & $\begin{array}{l}\text { Brooks and } \\
\text { Corey } \\
(1964) \\
\text { model }\end{array}$ & $\begin{array}{c}\text { van } \\
\text { Genuchten } \\
(1980) \\
\text { variable } m, n\end{array}$ & $\begin{array}{l}\text { van } \\
\text { Genuchten } \\
(1980) \\
m=1-1 / n\end{array}$ & $\begin{array}{l}\text { Assouline } \\
\text { et al. } \\
(1998)\end{array}$ & $\begin{array}{l}\text { This study } \\
\text { (artificial neural network) }\end{array}$ \\
\hline Beit Netofa clay soil & 1006 & 0.044 & 0.0245 & 0.034 & 0.017 & 0.012 \\
\hline Rideau clay loam & 3101 & 0.02 & 0.019 & 0.033 & 0.028 & 0.016 \\
\hline Touchet silt loam & 3304 & 0.015 & 0.0105 & 0.045 & 0.034 & 0.019 \\
\hline Pachappa loam & 3403 & 0.055 & 0.051 & 0.0653 & 0.030 & 0.058 \\
\hline Rubicon sandy loam & 3501 & 0.015 & 0.0148 & 0.05 & 0.038 & 0.025 \\
\hline Pachappa fine sandy clay & 3503 & 0.034 & 0.03 & 0.0346 & 0.041 & 0.036 \\
\hline Sable de riviere & 4118 & - & 0.00118 & 0.00118 & 0.042 & 0.0074 \\
\hline
\end{tabular}

ing for soils for which the difference between the maximum and the minimum values of suction was extremely large. For instance, the maximum and minimum values of the matric potential for the Pachappa loam soil were $319 \times 10^{4} \mathrm{~cm}$ and $10 \mathrm{~cm}$ of water. If the suction values were normalized by merely dividing by the maximum value for such data, most values in the lower suction range clustered close to zero and this resulted in poor training. To overcome this problem, the suction values for such soils were first log transformed and then standardized to fall within the range of $0-1$. All available pairs of $\theta-h$ values were used to train the ANNs. These days, a number of software packages are available, commercially as well as in public domain, that can be used to train an ANN.

\section{Results and Discussion}

The values of the norm index obtained by Assouline et al. (1998) and in this study are given in Table 2. Also included in Table 2 are norm values obtained by applying the van Genuchten (1980) model assuming both independent $m$ and $n$ values, or the often assumed relationship $m=1-1 / n$ (van Genuchten and Nielsen 1985). Notice that the NI obtained using ANNs is significantly smaller (except for one soil) than those obtained by Assouline et al. (1998), with the results of van Genuchten being somewhat better than those by Assouline et al. (1998), especially when $m$ and $n$ are kept independent. These results are consistent with many other studies in which ANNs have been found to model the behavior of real-world data better than the empirical/regression models.

Fig. 3 graphs observed values of soil suction versus the moisture content for Rideau clay loam. Also shown in the figure are plots of the WRC curve obtained by using Eq. (3) (the parameters as given by Assouline et al. 1998 were used) and the ANN-based curve. Note that the input to the ANN model was only the suction head. It can be seen that the ANN curve is closer to the measured data points than the curve obtained using Eq. (3). The results of Table 2 also show that the performance of ANN is much better than the model of Assouline et al. (1998) and is comparable to the model of van Genuchten (1980) and Brooks and Corey (1964). Note that application of ANN requires that the data over the entire water retention range is available. Also, the Assouline et al. (1998) model cannot be extrapolated to lower pressure heads (higher suctions) without risking physically unrealistic retention values.

\section{Modeling of Hysteresis}

The second part of this study is concerned with describing the hysteretic behavior of soils using ANNs. This problem is more complex than modeling WRC because the shape of drying and wetting limbs of the curve is not the same. Additionally, an ANN has to be provided with enough data so that it can unambiguously decide which curve to follow.

As before, the soil moisture data were normalized using Eq. (6), while the suction data were normalized by dividing by the maximum value. The root mean square error (RMSE) for the training/testing was calculated as

$$
\mathrm{RMSE}=\sqrt{\frac{1}{n} \sum_{i=1}^{n}\left(\theta_{a i}-\theta_{m i}\right)^{2}}
$$

where $n=$ number of data points, and $\theta_{a i}$ and $\theta_{m i}=$ actual and modeled values of the moisture content, respectively.

Initially, the ANN model was provided with two input values ( $h_{i}$ and $h_{i-1}$, i.e., the observed value of suction at the current and previous points) by reasoning that these would provide enough information for the model to determine which (main wetting or drying) branch to follow, and then correctly estimate the soil moisture. Note that in this case, the ANN training set consisted of all the available pairs of $h-\theta$ values for drying as well as wetting curves. The RMSE was quite small for the Rideau clay loam but was quite large for the Caribou silt loam. This experiment was followed up by including one more input in the ANN for $h_{i-2}$. This additional input should provide the ANN with the definite information whether the data is on the wetting limb or on the drying limb. As expected, inclusion of this additional input resulted in a large improvement in training results as reflected by a substantial reduction in the RMSE for both soils. The reduction in

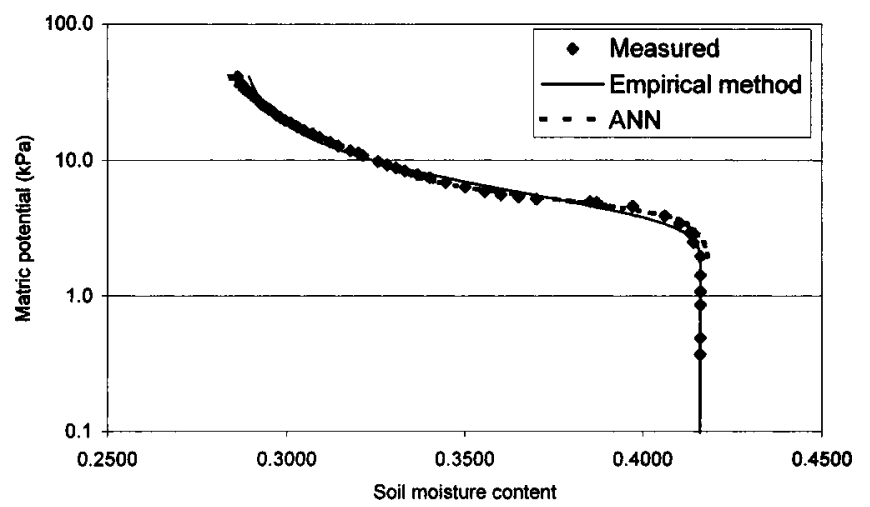

Fig. 3. Measured water retention data and curves obtained using artificial neural networks and empirical model of Assouline et al. (1998) 


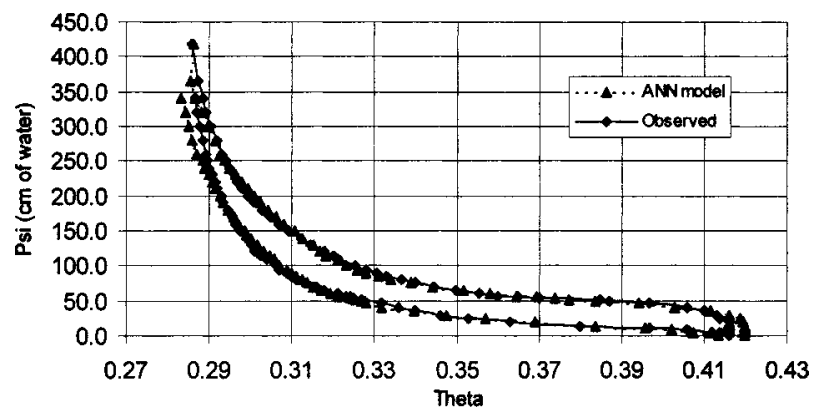

Fig. 4. Observed and artificial neural network modeled water retention curve for Rideau clay loam

RMSE was much larger for Caribou silt loam as compared to Rideau clay loam, with both soils now showing the same order of magnitude for RMSE.

Many other input combinations were tried. Another useful way to guide the ANN to the correct branch was found by including $h_{\text {init }}$ as input. This is the suction at the initial point of the $h-\theta$ curve. This will be the maximum suction value for the wetting curve and the minimum value for the drying curve. We found that including this information also led to a large reduction in the RMSE value. The observed and ANN-modeled curves for the two soils for this combination of inputs are given in Figs. 4 and 5. These figures show a good match between the observed and computed curves, except near saturation. Thus, a properly trained ANN which has four neurons in the input layer representing $h_{i}$, $h_{i-1}, h_{i-2}$, and $h_{\text {init }}$ can satisfactorily reproduce the hysteretic behavior of a soil. The RMSE values for the various input combinations for the two soils are given in Table 3, Part I.

An attempt was also made to model the behavior of the scanning curves using ANN. In this case, the training set consisted of all the available pairs of $h-\theta$ values for main drying and wetting curves and data for one dry scan and one wet scan curve. The same combination of inputs, as earlier, was tried using the data of main drying and wetting as well as one drying scanning curve and one wet scanning curve. The results are given in Part II of Table 3. These results also show that the training RMSE was quite small when the inputs were $h_{i}, h_{i-1}, h_{i-2}$, and $h_{\text {init }}$.

Since data for more than one dry and wet scan curves for each soil were available, the goodness of the ANN was tested using the data of a dry scan and wet scan that was not used in the training. The results are given in Parts III and IV of Table 3 and Figs. 6 and 7. Evidently, the match between observed and computed dry and wet scan curves was good for Soil 3101 while it was not that good for the wet scan curve of Soil 3301. However, the shape of a scan curve is significantly influenced by the drying and wetting history

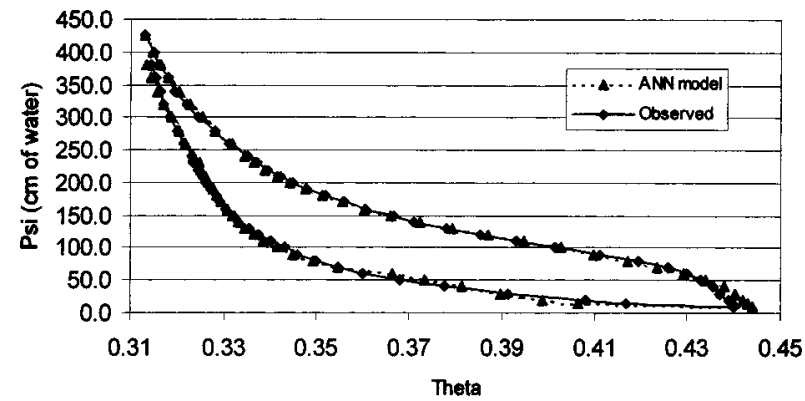

Fig. 5. Observed and artificial neural network modeled water retention curve for Caribou silt loam
Table 3. Results of Artificial Neural Network Modeling: Root Mean Square Error (RMSE) for Soils for Different Input Vectors

\begin{tabular}{lcc}
\hline & \multicolumn{2}{c}{ RMSE } \\
\cline { 2 - 3 } Inputs & $\begin{array}{c}\text { Rideau clay loam } \\
\text { (No. 3101) }\end{array}$ & $\begin{array}{c}\text { Caribou silt loam } \\
(\text { No. 3301) }\end{array}$ \\
\hline \multicolumn{3}{c}{ I. Main drying and wetting data } \\
\hline$h_{i}, h_{i-1}$ & 0.006073 & 0.015291 \\
$h_{i}, h_{i-1}, h_{i-2}$ & 0.004783 & 0.004679 \\
$h_{i}, h_{i-1}, h_{\text {init }}$ & 0.008849 & 0.002032 \\
$h_{i}, h_{i-1}, h_{i-2}, h_{\text {init }}$ & 0.002466 & 0.002431 \\
\hline \multicolumn{1}{c}{ II. Main drying, wetting, and dry/wet scan data } \\
\hline$h_{i}, h_{i-1}, h_{\text {init }}$ & 0.013179 & 0.009246 \\
$h_{i}, h_{i-1}, h_{i-2}, h_{\text {init }}$ & 0.007314 & 0.003045 \\
\hline \multicolumn{3}{c}{ III. Test-dry scan curve } \\
\hline$h_{i}, h_{i-1}, h_{\text {init }}$ & 0.008189 & 0.02348 \\
$h_{i}, h_{i-1}, h_{i-2}, h_{\text {init }}$ & 0.006781 & 0.00608 \\
\hline & IV. Test-wet scan curve \\
\hline$h_{i}, h_{i-1}, h_{\text {init }}$ & 0.004979 & 0.04636 \\
$h_{i}, h_{i-1}, h_{i-2}, h_{\text {init }}$ & 0.008485 & 0.02063 \\
\hline
\end{tabular}

of the soil and when seen with this perspective, coupled with data errors, etc., the results appear to be very good and useful.

Limited numerical experiments to determine the impact of missing data and errors in data on ANN training were also made. In the first case, three or four successive data points were deleted from a segment of WRC and the ANN was trained. We found that for our data sets RMSE changed by less than $0.5 \%$. Introduction of $10 \%$ error in some data points of the training set also led to about the same change in RMSE. As the ANN tries to generalize the data behavior, it assumes the behavior shown by the erroneous data set to be the true behavior of WRC and therefore, RMSE does not change significantly.

It may be added that when the values of norm index and RMSE are small, these indices may not be adequate to differentiate among the models and additional criteria, such as model reliability need to be used.

\section{Conclusions}

The WRC of a soil was modeled by an ANN using the measured data of soil moisture content and suction. The ANN used in this

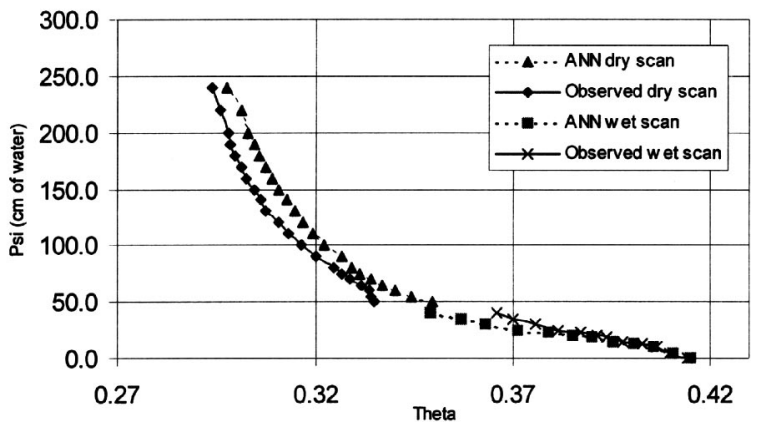

Fig. 6. Observed and artificial neural network modeled scan curves for Rideau clay loam 


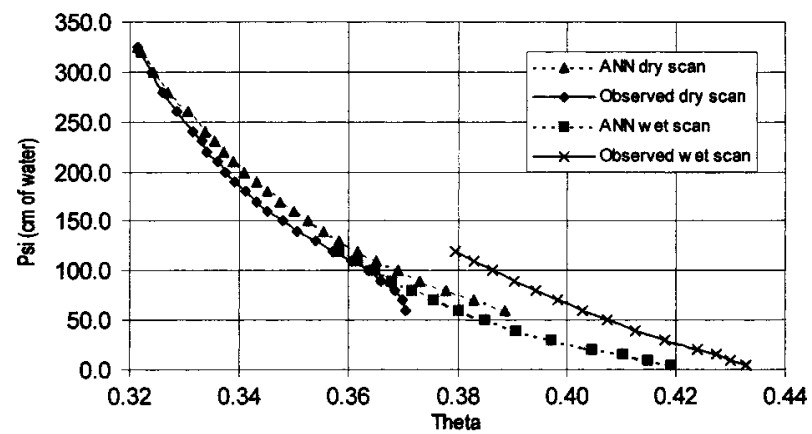

Fig. 7. Observed and artificial neural network modeled scan curves for Caribou silt loam

study was a three-layer feed-forward architecture. It was found that the ANN with only suction head data as input was able to describe WRC better than did the empirical model of Assouline et al. (1998) and the performance was comparable to the van Genuchten (1980) model. More importantly an ANN, whose inputs were the current and previous values of suction heads, was found to reproduce the hysteretic behavior and scan curves in a satisfactory manner. This application of ANN as a fitting tool should be useful in soil-moisture modeling.

\section{Notation}

The following symbols are used in this paper:

$h=$ soil suction (or matric or pressure head);

$h_{L}=$ soil suction corresponding to very low water content;

$\theta=$ volumetric water content of soil;

$\theta_{L}=$ very low water content of soil at which hydraulic conductivity becomes negligible;

$\theta_{r}=$ residual (volumetric) water content of soil; and

$\theta_{s}=$ saturated (volumetric) water content of soil.

\section{References}

American Society of Civil Engineers (ASCE) Task Committee on Application of Artificial Neural Networks in Hydrology. (2000a). "Artificial neural networks in hydrology. I: Preliminary concepts." J. Hydrologic Eng., 5(2), 115-123.

American Society of Civil Engineers (ASCE) Task Committee on Application of Artificial Neural Networks in Hydrology. (2000b). "Artificial neural networks in hydrology. II: Hydrological applications." $J$. Hydrologic Eng., 5(2), 124-137.

Assouline, S., Tessier, D., and Bruand, A. (1998). "A conceptual model of the soil water retention curve." Water Resour. Res., 34(2), 223 231.

Birikundavyi, S., Labib, R., Trung, H. T., and Roisselle, J. (2002). "Performance of neural networks in daily streamflow forecasting." $J$. $\mathrm{Hy}$ drologic Eng., 7(5), 392-398.

Brooks, R. H., and Corey, A. T. (1964). "Hydraulic properties of porous media." Hydrology Paper 3, Colorado State Univ., Fort Collins, Colo.
Dawson, C. W., and Wilby, R. (1998). "An artificial neural network approach to rainfall-runoff modelling." Hydrol. Sci. J., 43(1), 47-66.

Efron, B., and Tibshirani, R. J. (1993). "An introduction to the bootstrap." Monographs on statistics and applied probability 57, Chapman \& Hall, London.

Everett, D. H. (1955). "A general approach to hysteresis 4-An alternative formulation of the domain model." Trans. Faraday Soc., 51, $1551-1557$.

Govindaraju, R. S., and Rao, A. R. (2000). Artificial neural networks in hydrology, Kluwer Academic, Dordrecht, The Netherlands.

Hillel, D. (1971). Soil and water: Physical principles and processes, Academic, New York.

Hsu, K.-L., Gupta, H. V., and Sorooshian, S. (1995). "Artificial neural network modeling of the rainfall-runoff process." Water Resour. Res., 31(10), 2517-2530.

Jain, S. K., Das, A., and Srivastava, D. K. (1999). "Application of ANN for reservoir inflow prediction and operation." J. Water Resour. Plan. Manage., 125(5), 263-271.

Kastanek, E. J., and Nielsen, D. R. (2001). "Description of soil water characteristics using cubic spline interpolation." Soil Sci. Soc. Am. J., 65(2), 279-283.

Koekkoek, E. J. W., and Booltink, H. (1999). "Neural network models to predict soil water retention." Eur. J. Soil Sci., 50, 489-495.

Kumar, M., Raghuwanshi, N. S., Singh, R., Wallender, W. W., and Pruitt, W. O. (2002). "Estimating evapotranspiration using artificial neural network." J. Irrig. Drain. Eng., 128(4), 224-233.

Leij, F. J., Russell, W. B., and Lesch, S. M. (1997). "Closed-form expressions for water retention and conductivity data." Ground Water, 35(5), $848-853$.

Marquardt, D. W. (1963). "An algorithm for least-squares estimation of nonlinear parameters." J. Soc. Ind. Appl. Math., 11, 431-441.

Minasny, B., and McBratney, A. B. (2002). "The neuro-m method for fitting neural networks parametric pedotransfer functions." J. Soil Soc. Am., 66, 353-361.

Minns, A. W., and Hall, M. J. (1996). "Artificial neural networks as rainfall runoff models." Hydrol. Sci. J., 41(3), 399-418.

Mualem, Y. (1974). A catalogue of the hydraulic properties of unsaturated soils, Technion, Israel Institute of Technology, Haifa, Israel.

Pachepsky, Y. A., Timlin, D., and Varallyay, G. (1996). "Artificial neural network to estimate soil water retention from easily measurable data." Soil Sci. Soc. Am. J., 60, 727-733.

Prunty, L., and Casey, F. X. M. (2002). "Soil water retention curve using a flexible smooth function." Vadose Zone J., 1, 179-185.

Raman, H., and Sunilkumar, N. (1995). "Multivariate modeling of water resources time-series using artificial neural networks." Hydrol. Sci. J., 40(2), 145-163.

Schaap, M. G., and Leij, F. L. (2000). "Improved prediction of unsaturated hydraulic conductivity with the Mualem-van Genuchten model." Soil Sci. Soc. Am. J., 64, 843-851.

Schaap, M. G., Leij, F. L., and van Genuchten, M. Th. (1998). "Neural network analysis for hierarchical prediction of soil hydraulic properties." Soil Sci. Soc. Am. J., 62, 847-855.

Topp, G. C. (1971). "Soil water hysteresis in silt loam and clay loam soils." Water Resour. Res., 7(4), 914-920.

van Genuchten, M. Th. (1980). "A closed-form equation for predicting the hydraulic conductivity of unsaturated soils." Soil Sci. Soc. Am. J., 44, 892-898.

van Genuchten, M. Th., and Nielsen, D. R. (1985). "On describing and predicting the hydraulic properties of unsaturated soils." Ann. Geophys., 3(5), 615-628. 